\title{
REVISIÓN SOBRE LOS INSTRUMENTOS DE EVALUACIÓN DE LA DISCAPACIDAD EN PATOLOGÍA VESTIBULAR
}

\section{Review of instruments for evaluation of disability in vestibular pathology}

\author{
José Luis PARDAL-REFOYO'; Ángel BATUECAS-CALETRÍO² \\ SACYL. ${ }^{1}$ Complejo Asistencial de Zamora. Servicio de Otorrinolaringología. Zamora. España. IBSAL. Complejo \\ Asistencial de Salamanca. Servicio de Otorrinolaringología. Salamanca. España. \\ Correspondencia: jlpardal@usal.es
}

Fecha de recepción: 14 de enero de 2018

Fecha de aceptación: 27 de enero de 2018

Fecha de publicación: 30 de enero de 2018

Fecha de publicación del fascículo: 1 de junio de 2018

Conflicto de intereses: Los autores declaran no tener conflictos de intereses

Imágenes: Los autores declaran haber obtenido las imágenes con el permiso de los pacientes

Política de derechos y autoarchivo: se permite el autoarchivo de la versión post-print (SHERPA/RoMEO)

Licencia CC BY-NC-ND. Licencia Creative Commons Atribución-NoComercial-SinDerivar 4.0 Internacional

Universidad de Salamanca. Su comercialización está sujeta al permiso del editor

RESUMEN

Introducción y objetivo: Es necesario evaluar la repercusión subjetiva que la enfermedad vestibular tiene en el enfermo. Para esto se han desarrollado instrumentos de medida. La clasificación internacional de la funcionalidad de la OMS (CIF) indica evaluar cuatro niveles clave: las funciones corporales, la estructura corporal, la actividad y participación y los factores ambientales. Objetivo: recoger los instrumentos disponibles para evaluar la discapacidad según los parámetros de la CIF en el paciente con enfermedad vestibular mediante revisión bibliográfica. Método: Revisión narrativa. Búsqueda bibliográfica sistemática. Resultados: Se analizan los instrumentos de evaluación subjetiva del paciente (de medida de resultado informada por el paciente) recuperados en 5 artículos seleccionados tras una búsqueda bibliográfica sistemática. Conclusiones: Es importante recoger la percepción subjetiva del paciente mediante instrumentos de evaluación personal ya que es útil para evaluar la intervención terapéutica. Estos instrumentos deben ajustarse a los parámetros indicados en la CIF.

PALABRAS CLAVE vértigo; mareo; calidad de vida; vestibular; vestibulococlear; cuestionarios; psicometría tibular disease has on the patient. For this, measurement instruments have been developed. The WHO International Classification of Functioning (ICF) indicates the evaluation of four key levels: body functions, body structure, activity and participation, and environmental factors. Objective: to collect the instruments available to assess disability according to the parameters of the ICF in patient with vestibular disease through bibliographic review. Method: Narrative review. Systematic search. Results: The instruments of subjective patient-reported outcome recovered in 5 selected articles after a systematic bibliographic search are analyzed. Conclu- 


\section{INTRODUCCIÓN}

Los métodos objetivos de evaluación en pacientes con enfermedad vestibular frecuentemente no concuerdan con su experiencia subjetiva por lo que para evaluar la efectividad de las intervenciones vestibulares se requieren instrumentos apropiados validados para el uso en esta condición específica incluso adaptados a ciertos grupos de edad -como los ancianos, en los que los trastornos de equilibrio pueden llegar al $85 \%$ en edades superiores a 80 años con un mayor riesgo de caída- [1].

Los cuestionarios aportan información subjetiva sobre síntomas, estado funcional, percepción de la salud o calidad de vida del paciente independientemente de la interpretación externa del evaluador y son progresivamente más utilizados como medida de resultado en los estudios de intervención de rehabilitación vestibular puesto que existe gran variabilidad individual por lo que estos instrumentos puede ayudar a evaluar la evolución de un paciente en particular y a diferenciar grupos de pacientes [1].

CLASIFICACIÓN INTERNACIONAL DE LA FUNCIONALIDAD -CIF- (ICF, International Classification of Functioning) [1-3]

Para comprender por qué y cómo evaluar la repercusión y las limitaciones que la enfermedad vestibular puede provocar en el paciente necesitamos definir algunos aspectos clave (Tabla 1).

La Clasificación Internacional de la Funcionalidad (CIF) consta de dos componentes que se encuentran impedidos o limitados por la enfermedad: las funciones y estructuras corporales y las actividades y participación [3].

Esta idea viene a sustituir la anterior Clasificación Internacional de Deficiencias, Discapacidades y Minusvalías (CIDDM) de la Organización Mundial de la Salud (OMS) que proponía un esquema del proceso de la enfermedad y clasificaba las consecuencias que la enfermedad manifiesta en el paciente y en la sociedad con el esquema Enfermedad $\rightarrow$ Deficiencia $\rightarrow$ Discapacidad $\rightarrow$ Minusvalía [4] (Tabla 2).
Así la CIF evalúa cuatro niveles, aspectos o componentes clave sobre los que la enfermedad influye desde una perspectiva biopsicosocial [1,2]:

- Funciones corporales (body) -body level-.

- Estructuras corporales (structure).

- Actividad y participación (disability) -personal level-.

- Factores ambientales (environnment) -societal level-

Tabla 1. Clasificaciones y conceptos de la OMS sobre discapacidad [2]

\begin{tabular}{|l|l|}
\hline $\begin{array}{l}\text { Funciones cor- } \\
\text { porales }\end{array}$ & $\begin{array}{l}\text { funciones fisiológicas de los siste- } \\
\text { mas corporales (incluyendo las fun- } \\
\text { ciones psicológicas) }\end{array}$ \\
\hline $\begin{array}{l}\text { Estructuras cor- } \\
\text { porales }\end{array}$ & $\begin{array}{l}\text { partes anatómicas del cuerpo, tales } \\
\text { como los órganos, las extremida- } \\
\text { des y sus componentes }\end{array}$ \\
\hline Deficiencias & $\begin{array}{l}\text { problemas en las funciones o es- } \\
\text { tructuras corporales, tales como } \\
\text { una desviación o una pérdida }\end{array}$ \\
\hline Actividad & $\begin{array}{l}\text { desempeño o realización de una ta- } \\
\text { rea o acción por parte de un indivi- } \\
\text { duo }\end{array}$ \\
\hline $\begin{array}{l}\text { Limitaciones en } \\
\text { la actividad }\end{array}$ & $\begin{array}{l}\text { dificultades que un individuo puede } \\
\text { tener en el desempeño o realiza- } \\
\text { ción de las actividades }\end{array}$ \\
\hline Participación & $\begin{array}{l}\text { acto de involucrarse en una situa- } \\
\text { ción vital }\end{array}$ \\
\hline $\begin{array}{l}\text { Restricciones } \\
\text { en la participa- } \\
\text { ción }\end{array}$ & $\begin{array}{l}\text { problemas que el individuo puede } \\
\text { experimentar al involucrarse en si- } \\
\text { tuaciones vitales }\end{array}$ \\
\hline $\begin{array}{l}\text { Factores am- } \\
\text { bientales }\end{array}$ & $\begin{array}{l}\text { constituyen el ambiente físico, so- } \\
\text { cial y actitudinal en el que una per- } \\
\text { sona vive y conduce su vida }\end{array}$ \\
\hline
\end{tabular}

La enfermedad provoca alteraciones estructurales y funcionales que repercuten en las actividades cotidianas todo ello influido por factores ambientales y personales [2] (Figura 1):

1. Funcionamiento y discapacidad.

a. Funciones y estructuras corporales

i. Cambios en las funciones corporales

ii. Cambios en las estructuras corporales

b. Actividades y participación

i. Capacidad

ii. Desempeño/realización

2. Factores contextuales.

a. Factores ambientales

b. Factores personales 
Tabla 2. Conceptos de deficiencia, discapacidad y minusvalía.

\begin{tabular}{|l|l|}
\hline $\begin{array}{l}\text { DEFICIENCIA } \\
\text { gano }\end{array}$ & $\begin{array}{l}\text { Pérdida de la estructura o } \\
\text { función del órgano en- } \\
\text { fermo. }\end{array}$ \\
\hline & $\begin{array}{l}\text { Restricción o la pérdida } \\
\text { de la capacidad para rea- } \\
\text { lizar una actividad dentro } \\
\text { de la normalidad (conse- } \\
\text { cuencia de la deficiencia } \\
\text { del órgano). } \\
\text { «Impacto que las condi- } \\
\text { ciones crónicas o agudas } \\
\text { de la salud tienen sobre } \\
\text { el funcionamiento de sis- } \\
\text { temas corporales especí- } \\
\text { ficos y, de forma general, } \\
\text { sobre las habilidades de } \\
\text { las personas para actuar } \\
\text { de la forma necesaria, } \\
\text { usual, esperada o perso- } \\
\text { nalmente deseada en su } \\
\text { medio social» [4]. } \\
\text { «Brecha entre las capaci- } \\
\text { dades de una persona y } \\
\text { las demandas en su en- } \\
\text { torno físico y social» [4]. }\end{array}$ \\
$\begin{array}{l}\text { Restricción en las habili- } \\
\text { dades para realizar activi- } \\
\text { dades }\end{array}$ & $\begin{array}{l}\text { Situación de desventaja } \\
\text { social en la que se en- } \\
\text { cuentra el paciente como } \\
\text { consecuencia de la defi- } \\
\text { ciencia y la discapacidad. }\end{array}$ \\
\hline MINUSVALía & \\
Repercusión social &
\end{tabular}

Aplicado a la enfermedad vestibular la deficiencia es la alteración de la función del órgano enfermo - sistema vestibular- que provocará síntomas y signos - vértigo, inestabilidad, desequilibrio, mareo, hipoacusia, acúfenos, que podrán ser evaluados mediante la exploración física, métodos electrofisiológicos o estudios por imagen-, la incapacidad (discapacidad) es la reducción de la aptitud para mantener el equilibrio o la estabilidad del campo visual que imposibilitará realizar una actividad normal que puede demostrarse objetivamente mediante la observación, y la minusvalía que hace referencia a la desventaja social en la que se haya el individuo enfermo que puede medirse mediante escalas y cuestionarios (Figura 2) $[5,6]$.

Los instrumentos para evaluar la discapacidad, minusvalía y calidad de vida aplicados a la enfermedad vestibular deben evaluar los aspectos incluidos en la CIF (funciones y estructuras corporales y actividades y participación) [1-3].

Las deficiencias de los trastornos vestibulares y sus mediciones están bien establecidas (como por ejemplo pruebas calóricas, rotacionales, escalas analógicas visuales e informatizadas, posturografía dinámica), sin embargo las limitaciones de actividad y las restricciones de participación provocadas por trastornos vestibulares son en gran parte desconocidas debido a la ausencia de medidas especializadas en el área de actividades y participación (según la CIF) o bien a que los instrumentos disponibles mezclan elementos de funciones $y$ actividades corporales [3].

Hay muchas razones para medir las actividades y participación ya que son conceptos importantes en el cuidado de la salud y la rehabilitación que juegan un papel importante en la comprensión de la interacción entre el enfermo y los factores ambientales de su entorno, por lo que su evaluación individual puede permitir diseñar intervenciones personalizadas específicas [3].

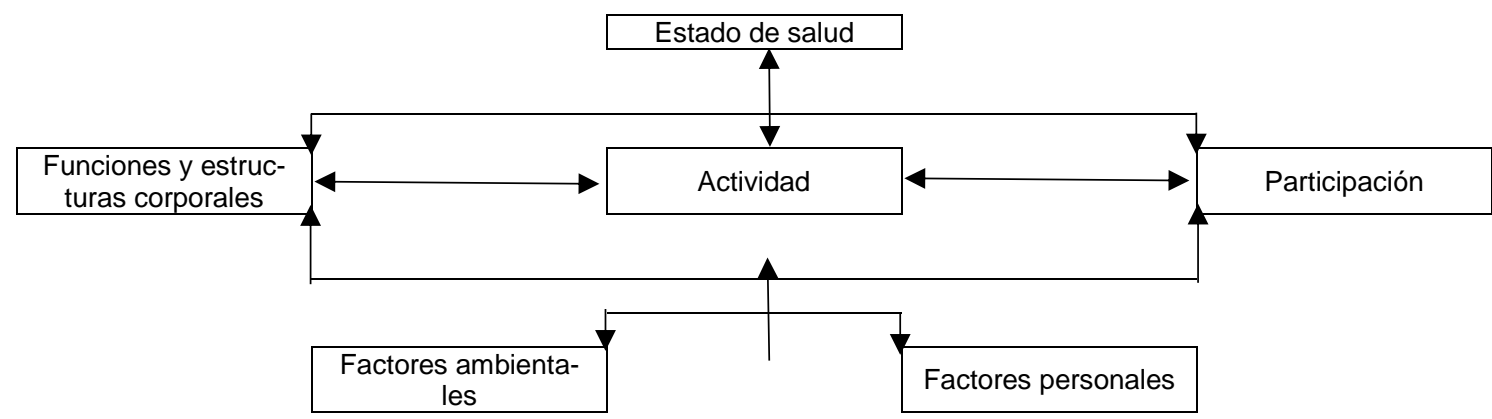

Figura 1. Factores que influyen sobre la actividad del individuo (tomado de Egea y cols. [2]) 


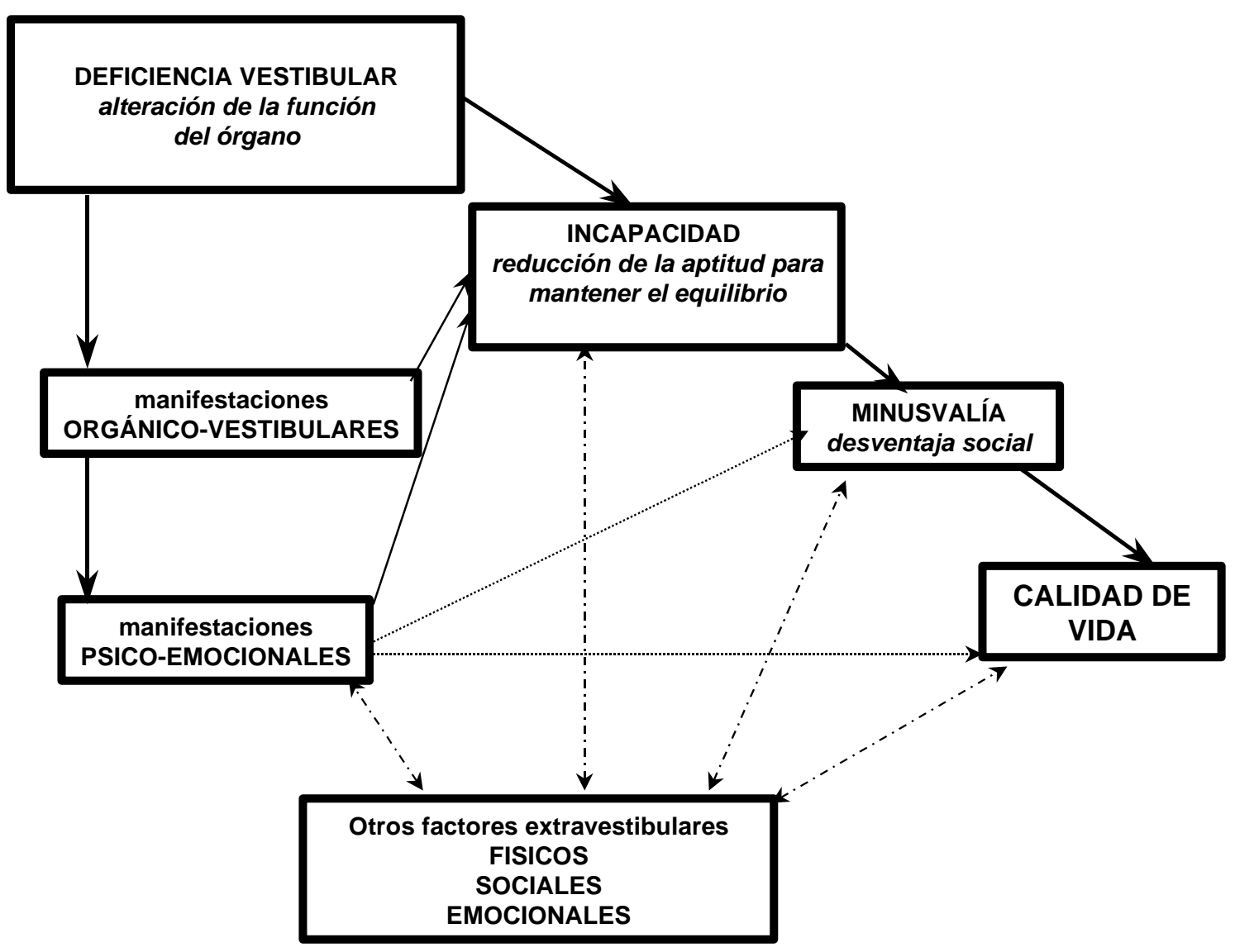

Figura 2. Deficiencia, minusvalía y calidad de vida en patología vestibular [6].

CONCEPTOS, TÉRMINOS MESH Y DEFINICIÓN (TABLA 3 - ANEXO 1)

El tema abordado es complejo y necesitamos establecer conceptos que nos permitan definir aquello que queremos medir para poder comparar los resultados obtenidos entre distintos autores, series o pacientes.

La Tabla 3 (Anexo 1) recoge los términos MeSH (https://www.ncbi.nlm.nih.gov/mesh/) y sus equivalentes DeCS en español (http://decs.bvs.br/) relacionados con la literatura que aborda los instrumentos de evaluación de la discapacidad, minusvalía o calidad de vida en patología vestibular.

ESCALAS Y CUESTIONARIOS UTILIZADOS EN LA EVALUACIÓN DE LA DISCAPACIDAD Y MINUSVALÍA DEL PACIENTE CON ENFERMEDAD VESTIBULAR

En la Tabla 4 (Anexo 2) y en la Tabla 5 se recogen algunos instrumentos utilizados para evaluar la repercusión funcional que, en el pa- ciente, provoca la enfermedad vestibular -modificación de las capacidades del individuo que padece patología vestibular (personalidad, memoria, ansiedad, depresión, inteligibilidad verbal, cuestionarios sobre salud en general y calidad de vida $=[7,8])$. Inicialmente los instrumentos utilizados iban orientados a evaluar la personalidad o los síntomas psicoafectivos (ansiedad, depresión, estrés). Progresivamente se fueron diseñando instrumentos específicos orientados a evaluar la repercusión funcional, discapacidad o la calidad de vida.

En general, los métodos pueden sistematizarse en escalas ordinales, escalas visuales analógicas, escalas y cuestionarios (de personalidad, de síntomas psicopatológicos o psicoafectivos y de sintomatología general) y específicos para la medición del impacto en patología vestibular.

En las escalas ordinales el observador asigna una puntuación o grado al estado del paciente (ejemplo: leve, moderado, intenso). Son las 
más utilizadas y aparecen en la mayoría de las publicaciones.

La escala visual analógica es una línea recta de diez centímetros que puede estar graduada o no graduada; en las formas no graduadas el paciente puede indicar la intensidad que, a su juicio, tiene la alteración del equilibrio, útil para utilizar en cada paciente como su propio control. En la forma graduada se da una puntuación o grado a una observación concreta. La escala visual permite una rápida conversión numérica y ser aplicada tanto a la sintomatología subjetiva como a la exploración del observador [9].

Las escalas y cuestionarios asignan una puntuación (que posteriormente será ponderada) a una frase (en el caso de las escalas) o a una pregunta (cuestionario); ambas pueden ser autoadministradas (el paciente lee y contesta sobre el test) o heteroadministradas (el investigador lee la frase o pregunta y el paciente contesta). La variedad de instrumentos que existen es enorme y están ampliamente difundidos [7,8].

Por último, se recogen también cuestionarios desarrollados y validados para registrar aspectos referentes a la incapacidad en pacientes con trastornos del equilibrio de origen vestibular con el fin de evaluar el impacto que sobre el individuo tiene la patología y la limitación que, sobre su autonomía produce [10-13].

El empleo de instrumentos específicos para evaluar la discapacidad y minusvalía en patología vestibular (handicap) es progresivo. En una revisión en PubMed (https://www.ncbi.nlm.nih.gov/) referido al periodo 1-1-1964 hasta 8-10-2017 se obtuvieron 10293 artículos de los cuales, 474 se refieren a discapacidad (minusvalía) con un incremento exponencial a partir de 1994). La publicación del DHI supuso un gran cambio en el enfoque de la evaluación de la minusvalía del paciente vestibular respecto a las aplicaciones que hasta 1990 estaban disponibles [13].

VALIDEZ DE LOS INSTRUMENTOS DE EVALUACIÓN Los instrumentos utilizados para evaluar discapacidad, minusvalía o calidad de vida en el paciente con enfermedad vestibular deben garantizar su validez en su elaboración, en su consistencia interna (test-retest, correlación a Cronbach), en su validez externa y en caso de la adaptación a otros idiomas haber realizado la traducción, aplicación y la traducción inversa $[14,15]$. Se deben tener en consideración el número de ítems, los métodos utilizados para desarrollar el instrumento, la población en la que se validó el instrumento y la fiabilidad testretest [1].

Tabla 5. Clasificación de los métodos empleados para la medición de la incapacidad en patología vestibular [4].

Instrumentos para evaluar calidad de vida o de discapacidad (DHI, VHQ, VADL, FSL, ABC y CEMPV)

Dizziness Handicap Inventory (DHI)

SF-36, SF-12

Ecalas de Cohen (VADL)

Vertigo Handicap Questionnaire (VHQ)

Functional Level Scale (FLS)

Activities-specific Balance Confidence (ABC)

Cuestionario para la Evaluación de la Minusvalía en Patología Vestibular (CEMPV)

Instrumentos para evaluar síntomas (VSS y EEV)

Vertigo Symptom Scale (VSS)

European Evaluation of Vertigo (EEV)

Instrumentos mixtos* (VDI, UCLA-DQ, DFI y MD-POSI) Vertigo, Dizziness, Imbalance (VDI)

Cuestionario del vértigo de la Universidad UCLA (UCLADQ)

Dizzy Factor Inventory (DFI)

Ménière's Disease Patient-Oriented Severity In$\operatorname{dex}(\mathrm{MDPOSI})$

* analizan síntomas y calidad de vida o discapacidad

Para evaluar la calidad de un instrumento puede ser útil la lista de verificación ERIQA (European Regulatory Issues on Quality of Life Assessment Working Group) que se recoge en la Tabla 6 [15,16] o EMPRO (Evaluating Measures of Patient Reported Outcomes) que es una herramienta desarrollada por BiblioPRO para la evaluación estandarizada de los instrumentos de resultados centrados en los pacientes (PRO, Patient Reported Outcomes) compuesta por 39 items que miden 8 atributos -modelo conceptual y de medición (7 items), adaptaciones culturales y lingüísticas (3 items), fiabilidad (8 items), validez (6 items), sensibilidad al cambio (3 items), interpretabilidad (3 items), carga de administración (7 items) y modos de administración alternativos (2 items) - [17].

Una limitación fundamental al realizar un estudio como éste, que es una limitación de la evaluación del paciente con vértigo en general, es que, al estudiar la repercusión funcional del vértigo en los pacientes, no existen cuestionarios adaptados a las diferentes patologías que 
cursan con vértigo. Existen patologías episódicas o únicas y existen patologías crónicas cuya repercusión en la vida de los enfermos es diferente.

Tabla 6. Lista de verificación utilizada para evaluar la validez de los cuestionarios (ERIQA, European Regulatory Issues on Quality of Life Assessment Working Group) $[15,16]$.

1. Generación de elementos y modelo conceptual subyacente al desarrollo del cuestionario Nivel de entrada de los pacientes

2. Descripción y adecuación de la población involucrada en los diferentes pasos de validación

3. Tamaño de la población involucrada en los diferentes pasos de validación

4. Descripción del cuestionario (cantidad de elementos y dimensiones)

5. Escala (opciones de respuesta) y puntuación

6. Recuperación del período (período al que deben relacionarse las respuestas)

7. Proceso de reducción del artículo (puede implicar la distribución de la respuesta, opciones, validez de contenido, opinión de expertos y análisis psicométricos: es decir, análisis factorial)

8. Consistencia interna (nivel de correlación de los ítems en una dimensión) *

9. Test-retest (estabilidad de las puntuaciones a lo largo del tiempo cuando la condición del paciente se considera estable) *

10. Validez del contenido (los elementos y las opciones de respuesta son relevantes y comprensivo de las dimensiones)

11. Validez estructural (análisis factorial o similar para apoyar la estructura de escala hipotética: es decir, la combinación de elementos en dimensiones) $\dagger$

12. Validez discriminatoria (capacidad del cuestionario para discriminar los pacientes de acuerdo con ciertas características, por ejemplo, la gravedad de la enfermedad) $\dagger$

13. Validez convergente (correlaciones del cuestionario con una escala conocida como de referencia o que se supone que mide más o conceptos menos similares) † 14. Validez predictiva (la futura evolución de la enfermedad puede predecirse por cambios de puntuación del cuestionario) $t$

15. Capacidad de respuesta (capacidad de detectar cambios)

* Consistencia interna y fiabilidad del cuestionario de la prueba-reprueba.

† Validez estructural, discriminante y convergente es parte de la validez del constructo que evalúa la conformidad con el modelo conceptual de la relación entre artículos y dimensiones

Las puntuaciones de cada apartado oscilan entre 0 (malo) y 4 (bueno).

La puntuación total puede expresarse en porcentajes en un rango de 0 (malo) a 100 (bueno).

La inestabilidad (más que el vértigo), la cronicidad de los síntomas, la frecuencia de las crisis y el conflicto visual parecen ser los síntomas más trascendentes (en lo que a calidad de vida se refiere) de los pacientes con patología vestibular [18].

El objetivo de este artículo es recoger los instrumentos disponibles para evaluar la discapacidad según los parámetros de la CIF en el paciente con enfermedad vestibular mediante revisión bibliográfica.

\section{MATERIAL Y MÉTODO}

Se realizaron búsquedas en las bases de datos PubMed y Cochrane Library con los descriptores y estrategias que se muestran en la Figura 3.

Criterios de selección: artículos de revisión sistemática (Systematic Reviews) que se referían a la utilización de cuestionarios o escalas para la evaluación de la discapacidad (Disability Evaluation) aplicadas a pacientes con enfermedades vestibulares (vestibular diseases), en idioma inglés o español, sin restricción de edad en el periodo 1-1-2007 a 8-10-2017.

Criterios de exclusión: ensayos clínicos (de 38 ensayos publicados), metanálisis (2 publicados) y artículos de investigación específicos de un diagnóstico o intervención específica.

Se seleccionaron 19 artículos que cumplían los criterios tras la lectura del resumen, de ellos 5 artículos cumplían los criterios de inclusión para el análisis cualitativo $[1,3,16,19,20]$.

\section{RESULTADOS}

Los instrumentos de medida de resultado informado por el paciente (PRO, Patient Reported Outcomes) se definieron como aquéllos que recogen la experiencia subjetiva del paciente, como los síntomas, su estado funcional y sus percepciones de salud y la calidad de vida [1]. En la Tabla 7 (Anexo 3) se exponen las características más importantes de los artículos revisados respecto a su metodología y principales resultados.

Los instrumentos más utilizados se recogen en la Tabla 8 [1].

INSTRUMENTOS QUE EVALÚAN CALIDAD DE VIDA Y MINUSVALÍA (HANDICAP)

\section{DHI Dizziness Handicap Inventory [13]}

Es un cuestionario autoadministrado con 25 items agrupados en tres dimensiones (emocional, funcional y orgánica) con correlación con ocho dimensiones del cuestionario genérico 
S36 [16]. Aunque ha sido cuestionado metodológicamente, actualmente es el cuestionario más utilizado [19] de referencia, traducido y adaptado a diversos idiomas no siempre de forma adecuada lo que limita la comparabilidad de los resultados [16]. Investigaciones posteriores han hallado en estudios factoriales factores diferentes a los inicialmente indicados por Jacobson (como, por ejemplo: «discapacidad en actividades de la vida diaria», "evitación fóbica» y "dificultades posturales», «discapacidad vestibular», «minusvalía vestibular» y «discapacidad visuo-vestibular») [16].
Es el cuestionario que más literatura ha generado. Sin embargo, tiene el inconveniente de que las dimensiones descritas originalmente por los autores no se corresponden con las identificadas en los análisis factoriales en estudios posteriores $[4,16]$ y no se ajusta a las orientaciones de la CIF; no evalúa los síntomas auditivos ni neurovegetativos, ni la repercusión en el cuidado personal [19]. Está adaptada y publicada la versión en español [21].

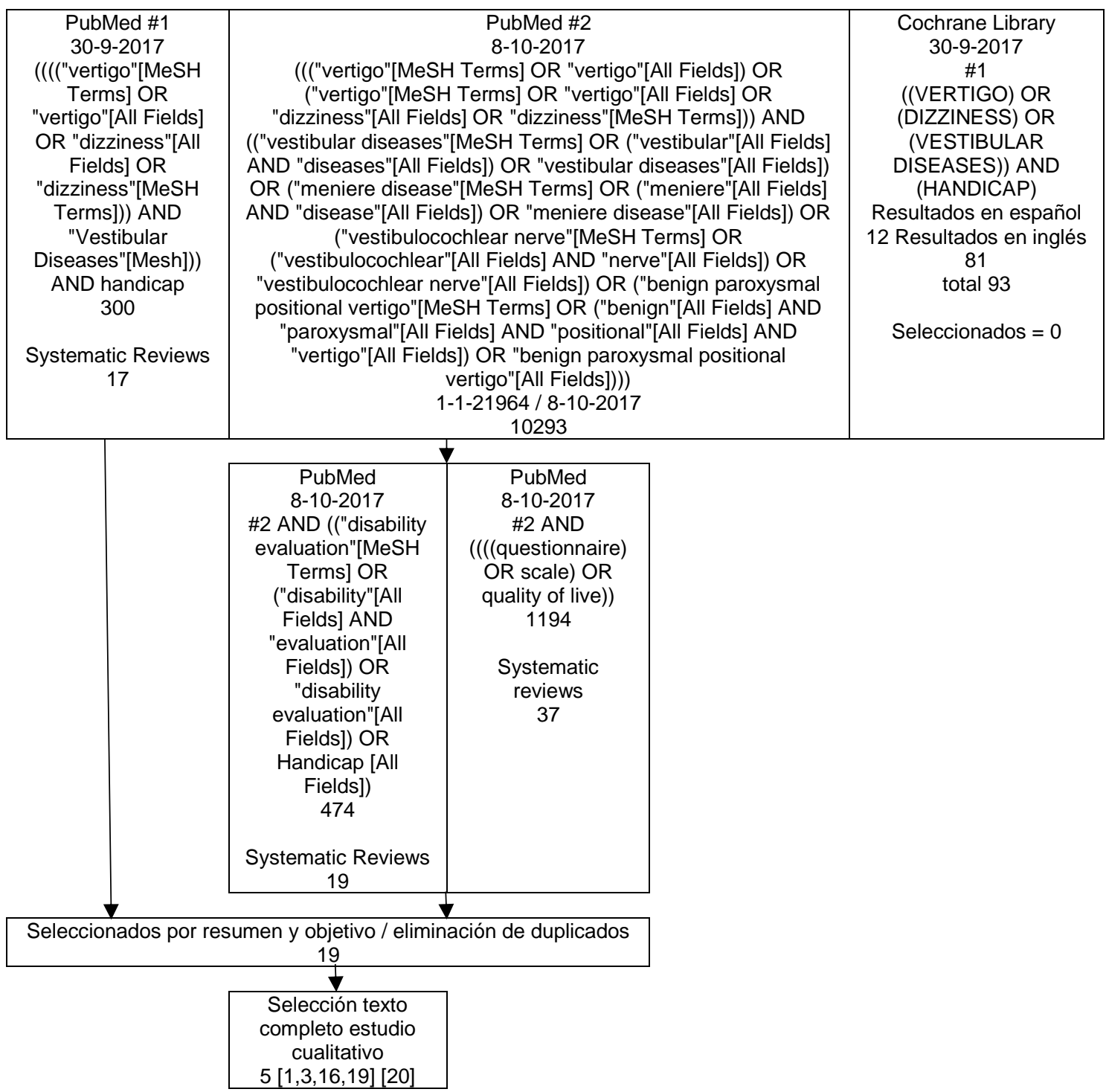

Figura 3. Método. Búsqueda bibliográfica sobre instrumentos para la evaluación de la discapacidad en pacientes con enfermedades vestibulares. 
Tabla 8. Instrumentos más utilizados en los artículos seleccionados en la revisión de Fong, 2015 [1].

\begin{tabular}{|l|l|}
\hline Activities of Daily Living Questionnaire & Patient Specific Functional Scale \\
Activities-specific Balance Confidence & Perceived Outcome Scale \\
Beck Depression Inventory (BDI) & Perceived Stress Scale \\
Centre for Epidemiological Studies Depression Scale & Perception of Dizziness Symptom \\
Chambless Mobility Inventory & Quantification of dizziness \\
Composite Score & Questionnaire, customized \\
Confidence in Everyday Activities Questionnaire & Rating Scale \\
Diary Registration & Repeatable Battery for the Assessment of Neuropsycho- \\
Disability Rating Scale & logical Status \\
Disability Score & Spielberger's Trait Anxiety Inventory \\
Dizziness Beliefs Questionnaire & Subjective Stability Evaluation \\
Dizziness Factor Inventory & Symptom Outcome Score \\
Dizziness Handicap Inventory & Symptom Severity Score (vertigo; dizziness; nausea; \\
European Quality of Life Questionnaire & Mann's test; Stepping test; Hallpike manoeuvre) \\
Falls & Symptoms \\
Global Improvement Rate & Tinetti Fall Risk \\
Hamilton Anxiety Scale & UCLA Dizziness Questionnaire \\
Handicap & VAS for Anxiety \\
Hospital Anxiety and Depression Scale & Vertigo Coping Questionnaire \\
Human Activity Profile & Vertigo Dizziness Imbalance Questionnaire \\
Medical Short-form & Vertigo Handicap Questionnaire \\
Modified Fatigue Impact Scale & Vertigo Symptom Index \\
Motion Sickness Questionnaire - Short form & Vertigo Symptom Scale, short form \\
Motor sensitivity & Vestibular Activities of Daily Living \\
Patient Enabler Instrument & Vestibular Rehab Benefit Questionnaire \\
& VHT Test Battery \\
\hline
\end{tabular}

VADL Vestibular Disorders of Daily Living Scale [22] Evalúa el impacto que la enfermedad vestibular tiene en las actividades de la vida diaria a través de 28 items agrupados en tres dimensiones (funcional -autocuidado y actividades personales-, deambulación -caminar y subir escaleras-e instrumental -gestión del hogar y actividades de ocio-).

EI VADL ofrece algunas deficiencias respecto a su escasa capacidad discriminante (entre patologías agudas o crónicas) o su reproducibilidad por lo que no parece muy específico para el vértigo [16].

ABC Activities-specific Balance Confidence [23] Mediante 16 items especifica las dificultades cotidianas de las personas mayores y el miedo a la caída. No es un cuestionario específico para el estudio del vértigo y procede de otra escala (Falls Efficacy Scale) por lo que no está claro si aporta mejor información [16].

\section{VHQ Vertigo Handicap Questionnaire [11]}

Es un cuestionario que consta de 22 items que reflejan las consecuencias incapacitantes de la enfermedad vestibular (orgánicas, en las actividades cotidianas, en la vida social y en el ocio). Probablemente es uno de los cuestionarios más prometedores, pero precisa la aplicación a mayor número de pacientes [16].

\section{CUESTIONARIOS MIXTOS}

VDI Vertigo, Dizziness, Imbalance Questionnaire [24]

Es un cuestionario con 36 items que miden la sensación de mareo e inestabilidad y sus consecuencias psicosociales. Su desarrollo empírico plantea dudas sobre la validez de las escalas [16].

UCLA-DQ UCLA Dizziness Questionnaire [25] El cuestionario contiene cinco elementos que evalúan la frecuencia y la gravedad de los mareos, el impacto en la actividad diaria, calidad de vida y miedo al mareo.

El nivel de correlación no se publicó en los trabajos iniciales, pero en estudios posteriores se demostró relación entre la frecuencia, miedo al mareo y calidad de vida, y entre la frecuencia y la gravedad del mareo y los otros tres elementos del cuestionario que variaron significativamente con el diagnóstico: la mayor discapacidad se observó en trastornos psicógenos, y la menor discapacidad en el vértigo posicional benigno $[21,26]$. Ha sido adaptado al castellano [21].

DFI Dizzy Factor Inventory [25]

Recoge 44 elementos en tres dimensiones (síntomas, percepción del paciente y nivel de actividad). 
Es un cuestionario que precisa estudios de comprobación de la validación [16].

VAP Vestibular Activities and Participation [3] Recoge 35 items en 5 niveles. Se ajusta a los parámetros CIF.

\section{INSTRUMENTOS PARA EVALUACIÓN DE SÍNTOMAS}

\section{VSS Vertigo Symptom Scale}

La versión final incluye 27 elementos con una estructura de cuatro factores (síntomas, síntomas vegetativos, ansiedad y somatización). La versión traducida al español (mexicano) mantiene una estructura similar a la versión en inglés lo que refuerza su validez [16,27].

\section{EEV European Evaluation of Vertigo [28]}

Evalúa los cinco síntomas principales del síndrome vestibular: ilusión de movimiento, duración de la ilusión, intolerancia al movimiento, signos neurovegetativos e inestabilidad. Este cuestionario fue comparado con el cuestionario $\mathrm{AAO}-\mathrm{HNS}$, que evalúa la repercusión funcional del vértigo, y con la escala genérica SF36. Su fortaleza está en que evalúa sólo síntomas y su debilidad en la reproducibilidad de la fiabilidad de la recogida de datos de los evaluadores de los signos neurovegetativos, el nivel de concordancia entre las evaluaciones de los pacientes y de los médicos y que no hay correlación entre los cinco elementos de la escala (sobre todo entre la ilusión de movimiento y su duración) [16]. Aparte del registro autoadministrado del paciente tiene un registro que realiza el observador.

\section{CUESTIONARIO ESPECÍFICO PARA LA ENFERMEDAD DE MÉNIÈRE}

MD-POSI Meniere's Disease Patients-Oriented Severity Index [29]

Mediante 20 elementos (16 agrupados en cuatro dimensiones y otras cuatro individuales) recoge la severidad de los síntomas en cuatro dimensiones: los síntomas de los pacientes y estado funcional durante las crisis; los síntomas de los pacientes y estado funcional entre las crisis; repercusión de la enfermedad en la actividad diaria de los pacientes y repercusión de la enfermedad en el trabajo de los pacientes.

ESTÁNDARES: BRIEF ICF CORE SET [20] Las categorías del Brief ICF Vertigo Core Set se definen por un código alfanumérico que comienza con una letra minúscula que indica componente - «b» para las funciones corporales, «S» para las estructuras corporales, «d» para actividades y participación y «e» para factores ambientales-. Las letras son seguidas por un código numérico para el número de capítulo (un dígito, primer nivel), seguido del segundo nivel (dos dígitos), y el tercer y cuarto nivel (un dígito cada uno).

En la Tabla 9 se recogen las categorías del Brief ICF Core Set.

\section{CUESTIONARIOS EN CASTELLANO}

Existe una gran carencia de adaptación de cuestionarios de este tipo al castellano. El proceso es complejo y requiere un gran esfuerzo. Sin embargo, los beneficios que aporta la validación de los cuestionarios son grandes, no sólo desde el punto de vista de la investigación (para contrastar los resultados con series a nivel internacional) sino desde el punto de vista asistencial, sabiendo qué es lo que estamos midiendo. En la base de datos BiblioPRO están publicados cuestionarios desarrollados originalmente en castellano o adaptados y validados de otros idiomas al castellano (Tabla 10).

Tabla 10. Cuestionarios validados y desarrollados en castellano publicados en BiblioPRO

\begin{tabular}{|l|l|l|}
\hline Siglas & Título & País de origen \\
\hline VDI & $\begin{array}{l}\text { Cuestionario para los } \\
\text { Síntomas de Vértigo y } \\
\text { Mareo [31] }\end{array}$ & España \\
\hline DHI & $\begin{array}{l}\text { Versión española del } \\
\text { Dizziness Handicap In- } \\
\text { ventory [21] }\end{array}$ & $\begin{array}{l}\text { Estados Uni- } \\
\text { dos }\end{array}$ \\
\hline $\begin{array}{l}\text { UCLA- } \\
\text { DQ }\end{array}$ & $\begin{array}{l}\text { Versión española del } \\
\text { UCLA Dizziness Ques- } \\
\text { tionnaire [21] }\end{array}$ & $\begin{array}{l}\text { Estados Uni- } \\
\text { dos }\end{array}$ \\
\hline $\begin{array}{l}\text { BS / Ba- } \\
\text { lance } \\
\text { Scale }\end{array}$ & $\begin{array}{l}\text { Escala de Equilibrio de } \\
\text { Berg[31] }\end{array}$ & Canadá \\
\hline DHI-S & $\begin{array}{l}\text { Versión Española del } \\
\text { Dizziness Handicap In- } \\
\text { ventory - Versión redu- } \\
\text { cida [30] }\end{array}$ & Estados Uni- \\
dos
\end{tabular}

De los validados en castellano los más utilizados son el DHI y el UCLA-DQ [21]. Se validó una versión abreviada del DHI con 13 items (DHI-S [30]).

Otros cuestionarios validados en castellano son el VDI (cuestionario para síntomas de vértigo y mareo) - [31] que mide la calidad de vida 
relacionada con la salud, con 14 items sobre los síntomas y 22 sobre la calidad de vida- y, relacionado con este último, el cuestionario «Escala de equilibrio de Berg», pero no hemos encontrado el formulario en castellano en ninguna fuente.
Desarrollado en castellano también está disponible el CEMPV (Cuestionario para la evaluación de la minusvalía en patología vestibular) $[5,32,33]$.

Tabla 9. Categorías del Brief ICF Vertigo Core Set según la International Classification of Functioning, Disability and Health (ICF) [20].

\begin{tabular}{|c|c|}
\hline $\begin{array}{l}\text { b152 Emotional functions } \\
\text { b156 Perceptual functions } \\
\text { b210 Seeing functions } \\
\text { b215 Functions of structures adjoining the eye } \\
\text { b230 Hearing functions } \\
\text { b235 Vestibular functions } \\
\text { b240 Sensations associated with hearing and vestibular } \\
\text { function } \\
\text { b260 Proprioceptive function } \\
\text { b770 Gait pattern functions } \\
\text { d230 Carrying out daily routine } \\
\text { d410 Changing basic body position } \\
\text { d415 Maintaining a body position } \\
\text { d450 Walking } \\
\text { d455 Moving around } \\
\text { d460 Moving around in different locations } \\
\text { d469* Walking and moving, other specified and unspeci- } \\
\text { fied } \\
\text { d475 Driving } \\
\text { d640 Doing housework } \\
\text { (d840-d859) Work and employment } \\
\text { e110 Products or substances for personal consumption } \\
\text { e120 Products and technology for personal indoor and } \\
\text { outdoor mobility and transportation } \\
\text { e240 Light } \\
\text { e310 Immediate family } \\
\text { e355 Health professionals } \\
\text { e580 Health services, systems and policies } \\
\text { s110 Structure of brain } \\
\text { s120 Spinal cord and related structures } \\
\text { s260 Structure of inner ear } \\
\text { s410 Structure of cardiovascular system }\end{array}$ & $\begin{array}{l}\text { b152 Funciones emocionales } \\
\text { b156 Funciones de percepción } \\
\text { b210 Función visual } \\
\text { b215 Funciones de las estructuras contiguas al ojo } \\
\text { b230 Funciones auditivas } \\
\text { b235 Funciones vestibulares } \\
\text { b240 Sensaciones asociadas con la función auditiva y } \\
\text { vestibular } \\
\text { b260 Función propioceptiva } \\
\text { b770 Funciones de patrón de marcha } \\
\text { d230 Realización de la rutina diaria } \\
\text { d410 Cambiar la posición básica del cuerpo } \\
\text { d415 Mantenimiento de una posición corporal } \\
\text { d450 Caminando } \\
\text { d455 Moverse por } \\
\text { d460 Desplazarse por diferentes lugares } \\
\text { d469 * Caminar y moverse, otros especificados y no es- } \\
\text { pecificados } \\
\text { d475 Conducción } \\
\text { d640 Haciendo tareas domésticas } \\
\text { (d840-d859) Trabajo y empleo } \\
\text { e110 Productos o sustancias para consumo personal } \\
\text { e120 Productos y tecnología para movilidad y transporte } \\
\text { personal en interiores y exteriores } \\
\text { e240 Luz } \\
\text { e310 Familia inmediata } \\
\text { e355 Profesionales de la salud } \\
\text { e580 Servicios, sistemas y políticas de salud } \\
\text { s110 Estructura del cerebro } \\
\text { s120 Médula espinal y estructuras relacionadas } \\
\text { s260 Estructura del oído interno } \\
\text { s410 Estructura del sistema cardiovascular }\end{array}$ \\
\hline
\end{tabular}

\section{DISCUSIÓN}

El número de instrumentos $\mathrm{PRO}$ recogidos en el estudio de Fong es amplio (Tabla 8) [1] con gran heterogeneidad ya puesto de manifiesto en el estudio de Duracinsky [16] (disparidad de instrumentos y objetivos de evaluación, pocos pacientes evaluados y muestras no homogéneas). Por ello, para la selección de los instrumentos deben tenerse en cuenta los criterios CIF [1] con instrumentos como ERIQA [15] (Tabla 6), EMPRO (https://www.bibliopro.org/) o si incluyen las categorías del Brief ICF Core Set [20] (Tabla 9).

En general los instrumentos publicados tienen un espectro limitado y no tienen en cuenta todos los aspectos de la discapacidad asociados con el vértigo [20], sin embargo la evaluación de los síntomas de vértigo, mareo, calidad de vida y minusvalía es útil ya que estudios instrumentales no evalúan adecuadamente la repercusión en el paciente por lo que la utilización de instrumentos PRO para registrar síntomas y calidad de vida ofrecen valor añadido y permiten evaluar la eficacia de los tratamientos [16].

Parece lógico concentrar la investigación para obtener instrumentos estandarizados universales.

La validez de algunos cuestionarios es dudosa ya que los cuestionarios que evalúan calidad de vida deben seguir un procedimiento riguroso incluyendo generación de items, reducción de items, confiabilidad, validez (dejando 
un periodo de 1 a 3 semanas para realizar el retest) y capacidad de respuesta, así como traducción y adaptación cultural si es necesario; este proceso es iterativo, debe incluir cientos de pacientes y los pacientes reclutados en la validación deben ser representativos de los que padecen la enfermedad vestibular [16]. Además, es importante la escala de resultado de cada ítem que debe ser lo suficientemente amplia como para detectar cambios subjetivos pequeños - aumentando el número de categorías o incluyendo una escala visual analógica[16].

El instrumento más utilizado en la literatura y adaptado a diferentes idiomas y culturas es el DHI [19], pero el DHI presenta limitaciones indicadas por diversos autores (dimensiones diferentes a las definidas originalmente tras realizar análisis factoriales), defectos en la metodología de diseño y no seguir los criterios CIF [16]. En este sentido el Brief ICF Core Set [20] pretende establecer unas orientaciones mínimas en la evaluación de los pacientes con mareo o vértigo ajustado a las especificaciones CIF (función corporal, estructura corporal, actividades y participación y factores ambientales).

Recientemente se han desarrollado nuevos PRO como el Vestibular Activities and Participation (VAP) ajustado a los criterios CIF [16]. EI VAP evalúa las actividades y la participación de las personas con trastornos vestibulares de acuerdo con la CIF.

Varios estudios muestran una correlación débil o nula entre los síntomas y calidad de vida (evaluada por DHI, VADL, VSS) y los estudios instrumentales (prueba calórica y posturografía) [16]. La correlación con otras exploraciones vestibulares es variable y no evalúa los síntomas auditivos, neurovegetativos o aspectos funcionales como el autocuidado lo que limita la correlación con otros instrumentos de evaluación de discapacidad o calidad de vida [19] (Tabla 11).

Tabla 11. Correlación de los resultados de los instrumentos de evaluación de discapacidad o calidad de vida con otras exploraciones vestibulares [19].

\begin{tabular}{|c|c|c|}
\hline Alta correlación & Moderada correlación & Nula correlación \\
\hline \multicolumn{3}{|c|}{ Exploración vestibular } \\
\hline $\begin{array}{l}\text { - Posturografía dinámica computarizada } \\
\text { - Alcance funcional } \\
\text { - Electronistagmografía } \\
\text { - Índice dinámico de la marcha } \\
\text { - Prueba de impulso de la cabeza }\end{array}$ & 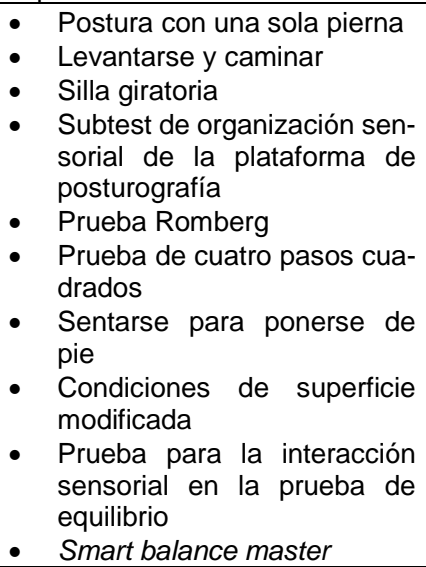 & 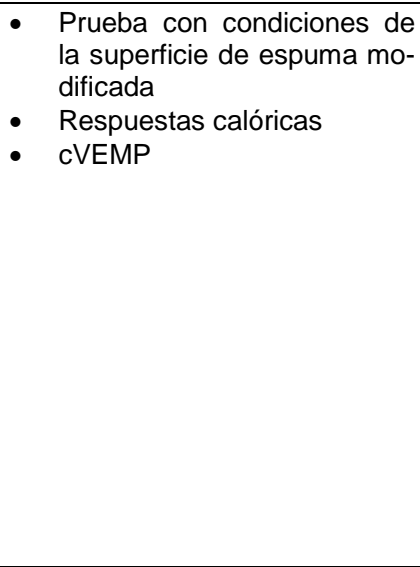 \\
\hline \multicolumn{3}{|c|}{ Otros instrumentos } \\
\hline $\begin{array}{ll}\text { - } & \text { Health Related Quality of Life (SF-36) } \\
\text { - } & \text { Hospital Anxiety and Depression Scale }\end{array}$ & \multicolumn{2}{|c|}{$\begin{array}{ll}\text { - } & \text { Activities Specific Balance Confidence Scale } \\
\text { - } & \text { Vestibular Disorders Activities of Daily Living Scale } \\
\text { - } & \text { Vertigo Symptom Scale } \\
\text { - } & \text { SFO Quality of Life Scale } \\
\text { - } & \text { Visual Vertigo Analogue Scale } \\
\text { - } & \text { Illness Perception Questionnaire } \\
\text { - } & \text { State Trait Anxiety Inventory }\end{array}$} \\
\hline
\end{tabular}

Los instrumentos PRO proporcionan una evaluación directa de la experiencia del paciente, que es lo que las intervenciones clínicas buscan mejorar en última instancia. Se ha demostrado que las medidas PRO reflejan mejor el estado de salud subyacente que las medidas objetivas, y se correlacionan bien con los 
resultados clínicos importantes, incluida la supervivencia y la satisfacción del paciente [1]. La valoración de la repercusión difiere entre pacientes y médicos como demostró el estudio de validación del UCLA-DQ ya que el médico tiende a subestimar los síntomas especialmente ante patologías no consideradas graves [16]. Indirectamente evalúan la calidad de vida que no puede deducirse exclusivamente de los síntomas ya que la correlación entre síntomas y calidad de vida es moderada (observada en los estudios con EEV y SF-36) [16]. Por tanto, es esencial el punto de vista del paciente [16]. Otro aspecto interesante es el periodo de reevaluación con un instrumento $\mathrm{PRO}$ ya que los recuerdos de los cambios sociales percibidos por el paciente pueden requerir más tiempo que para evaluar los cambios en los síntomas físicos que resultan del tratamiento médico (aquí el periodo de recuerdo debe ser corto).

\section{CONCLUSIONES}

El paciente con vértigo es un paciente que sufre y lo hace en una doble vertiente: la de la enfermedad en sí, con las limitaciones que conlleva, y la de la falta de solidaridad o empatía por el entorno, al ser una enfermedad «que no se ve».

Generalmente se tienden a minimizar los efectos de esta patología en la vida cotidiana de los enfermos, cuando la realidad es que su fisiopatología está íntimamente ligada a esas actividades, de forma que es imposible pensar que un paciente con vértigo no sufra sus consecuencias en su vida diaria, ya sea laboral o cotidiana.

Por ello, resulta necesario conocer la repercusión que la patología tiene en el enfermo y, hasta ahora, sólo podemos conocerlo a través de cuestionarios que aborden todos los ámbitos funcionales de los enfermos.

Es importante recoger la percepción subjetiva del paciente mediante instrumentos de evaluación personal ya que es útil para evaluar la intervención terapéutica.

La mayoría de los instrumentos desarrollados para evaluar minusvalía o calidad de vida no son muy específicos para aplicar en pacientes con vértigo o mareo ya que presentan limitaciones de validación y no se ajustan a los criterios de la CIF.

Recientemente se ha desarrollado el VAP Vestibular Activities and Participation que sigue dichos criterios.
Es necesario trabajar en la elaboración de instrumentos universales aplicados al paciente con patología vestibular.

Es necesario trabajar en la adaptación de instrumentos al español o desarrollo de instrumentos específicos en español.

\section{BIBLIOGRAFÍA}

1. Fong E, Li C, Aslakson R, Agrawal Y. Systematic review of patient-reported outcome measures in clinical vestibular research. Arch Phys Med Rehabil. 2015;96(2):357-65.

2. Egea García C, Sarabia Sánchez A Clasificaciones de la OMS sobre discapacidad. Boletín del Real Patron sobre Discapacidad. 2001;(50):15-30.

3. Alghwiri AA, Whitney SL, Baker CE, Sparto PJ, Marchetti GF, Rogers JC, et al. The Development and Validation of the Vestibular Activities and Participation Measure. Arch Phys Med Rehabil. 2012;93(10):1822-31.

4. Pérez Fernández N, Montes-Jovellar L, Boleas Aguirre MS. Calidad de vida en pacientes con enfermedad de Méniêre. En: Pérez Garrigues H, López Escámez JA, Morales Angulo C PFN, editor. Enfermedad de Ménière : desde las ciencias básicas hacia la medicina clínica. E.U.R.O.M.E.D.I.C.E. Ediciones Médicas, S.L; 2009. p. 173-9.

5. Pardal Refoyo JL. Comentarios sobre minusvalía en patología vestibular. Acta Otorrinolaringol Esp. 2001;52(2):169-70.

6. Pardal-Refoyo JL. Cuestionario para la Evaluación de la Minusvalía en Patología Vestibular Cuaderno de aplicación e interpretación. 2000. 29 p. Disponible en: https://www.bibliopro.org/buscador/3728/cue stionario-para-la-evaluacion-de-laminusvalia-en-patologia-vestibular-cempv

7. Teeling-Smith G. Measuring habits: a practical approach. John Wiley. Chihester; 1988.

8. Rosser R, Kind P, Williams A. Valuation of quality of life: some psychometric evidente. En: JONES-LEE M, editor. The value of life and society. Elservier-North Holland. Amsterdam; 1982. 
9. Pardal Jl, Beltran Ld. Medición de la intensidad e incapacidad en trastornos del equilibrio mediante escala visual. ORL-DIPS. 1994;20(2):78-81.

10. Yardley L, Verschuur C, Masson E, Luxon L, Haacke N. Somatic and psychological factors contributing to handicap in people with vertigo. Br J Audiol.1992;26(5):283-90.

11. Yardley L, Putman J. Quantitative analysis of factors contributing to handicap and distress in vertiginous patients: a questionnaire study. Clin Otolaryngol Allied Sci.1992;17(3):231-6.

12. Yardley L. Contribution of symptoms and beliefs to handicap in people with vertigo: a longitudinal study. Br J Clin Psychol. 1994;33 (Pt 1):101-13.

13. Jacobson GP, Newman CW. The development of the Dizziness Handicap Inventory. Arch Otolaryngol Head Neck Surg. 1990;116(4):424-7.

14. Chassany $O$, Sagnier $P$, Marquis $P$, Fulleton S AN. Patient Reported Outcomes and Regulatory Issues: the Example of Healthrelated Quality of Life - A European Guidance Document for the Improved Integration of HRQL Assessment in the Drug Regulatory Process. Drug Inf J. 2002;36(1):209-38.

15. Acquadro C, Conway K, Hareendran A, Aaronson N, European Regulatory Issues and Quality of Life Assessment (ERIQA) Group. Literature Review of Methods to Translate Health-Related Quality of Life Questionnaires for Use in Multinational Clinical Trials. Value Heal. 2008;11(3):50921.

16. Duracinsky M, Mosnier I, Bouccara D, Sterkers O, Chassany O, Working Group of the Société Française d'Oto-RhinoLaryngologie (ORL). Literature review of questionnaires assessing vertigo and dizziness, and their impact on patients' quality of life. Value Health. 2007;10(4):273-84.

17. Valderas JM, Ferrer M, Mendívil J, Garin O, Rajmil L, Herdman M, et al. Development of EMPRO: a tool for the standardized assessment of patient-reported outcome measures. Value Health. 2008;11(4):700-8.
18. Trinidad Ruiz G. Impacto en la calidad de vida y la situación laboral de los trastornos del equilibrio. En: Trinidad Ruíz G, editor. Definiciones e impacto del vértigo y sus síndromes relacionados (Ponencia oficial de la Sociedad Extremeña de Otorrinolaringología y Patología Cérvicofacial). Ed. Ergon; 2017. p. 207-25.

19. Mutlu B, Serbetcioglu B. Discussion of the dizziness handicap inventory. J Vestib Res. 2013;23(6):271-7.

20. Grill E, Bronstein A, Furman J, Zee DS, Müller M. International Classification of Functioning, Disability and Health (ICF) Core Set for patients with vertigo, dizziness and balance disorders. J Vestib Res. 2012; 22(5-6):26171.

21. Pérez N, Garmendia I, Martín E, GarcíaTapia R. Adaptación cultural de dos cuestionarios de medida de la salud en pacientes con vértigo. Acta Otorrinolaringol Esp. 2000;51(7):572-80.

22. Cohen HS, Kimball KT. Development of the vestibular disorders activities of daily living scale. Arch Otolaryngol Head Neck Surg. 2000;126(7):881-7.

23. Powell LE, Myers AM. The Activities-specific Balance Confidence (ABC) Scale. J Gerontol A Biol Sci Med Sci. 1995;50A(1):M28-34.

24. Prieto L, Santed R, Cobo E, Alonso J. A new measure for assessing the health-related quality of life of patients with vertigo, dizziness or imbalance: the VDI questionnaire. Qual Life Res. 1999;8(1-2):131-9.

25. Honrubia V, Bell TS, Harris MR, Baloh RW, Fisher LM. Quantitative evaluation of dizziness characteristics and impact on quality of life. Am J Otol. 1996;17(4):595-602.

26. Pérez N, Garmendia I, Martín E, Boleas MS, García-Tapia R. Instrumentos de medida de la salud en pacientes con vértigo. Acta Otorrinolaringol Esp. 2001;51(8):677-85.

27. Yardley L, Medina SM, Jurado CS, Morales TP, Martinez RA, Villegas HE. Relationship between physical and psychosocial dysfunction in Mexican patients with vertigo: a cross-cultural validation of the vertigo symptom scale. J Psychosom Res. 1999;46(1):63-74. 
28. Mègnigbêto CA, Sauvage JP, Launois R. The European Evaluation of Vertigo (EEV) scale: a clinical validation study]. Rev Laryngol Otol Rhinol (Bord). 2001;122(2):95102.

29. Murphy MP, Gates GA. Measuring the effects of Meniere's disease: results of the Patient-Oriented Severity Index (MD POSI) version 1. Ann Otol Rhinol Laryngol. 1999;108(4):331-7.

30. Lopez-Escamez JA, Viciana D, GarridoFernandez P. Impact of bilaterality and headache on health-related quality of life in Meniere's disease. Ann Otol Rhinol Laryngol. 2009;118(6):409-16.

31. Prieto L, Santed R, Cobo E, Alonso J. A new measure for assessing the health-related quality of life of patients with vertigo, dizziness or imbalance: the VDI questionnaire. Qual Life Res. 1999;8(1-2):131-9.

32. Pardal Refoyo JL, Beltrán Mateos LD, del Cañizo Alvarez A. Cuestionario para la Evaluación de la Minusvalía en Patología Vestibular (CEMPV). An Otorrinolaringol Ibero Am. 1998;25(6):547-64.

33. Pardal-Refoyo JL. Cuestionario para la Evaluacion de la Minusvalia en Patologia Vestibular: modelo, aplicacion y utilidad. ORL-DIPS. 1997;24(2):53-8.

34. Hinchcliffe R. Personality profile in Menière's disease. J Laryngol Otol. 1967;81(5):477-81 .

35. Brightwell DR, Abramson M. Personality characteristics in patients with vertigo. Arch Otolaryngol. 1975;101(6):364-6.

36. Stephens SD. Personality tests in Ménière's disorder. J Laryngol Otol. 1975;89(5):479-90.

37. Crary WG, Wexler M. Meniere's disease: a psychosomatic disorder? Psychol Rep. 1977;41(2):603-45.

38. Rigatelli M, Casolari L, Bergamini G, Guidetti G. Psychosomatic study of 60 patients with vertigo. Psychother Psychosom. 1984;41(2):91-9.

39. Hallam RS, Stephens SD. Vestibular disorder and emotional distress. J Psychosom Res. 1985;29(4):407-13.
40. Filipo R, Lazzari R, Barbara M, Franzese A, Petruzzellis MC. Psychologic evolution of patients with Menière's disease in relation to therapy. Am J Otol. 1988;9(4):306-9.

41. Coker NJ, Coker RR, Jenkins HA, Vincent KR. Psychological profile of patients with Menière's disease. Arch Otolaryngol Head Neck Surg. 1989;115(11):1355-7.

42. Grimm RJ, Hemenway WG, Lebray PR, Black FO. The perilymph fistula syndrome defined in mild head trauma. Acta Otolaryngol Suppl. 1989;464:1-40.

43. McKenna L, Hallam RS, Hinchcliffe R. The prevalence of psychological disturbance in neurotology outpatients. Clin Otolaryngol Allied Sci. 1991;16(5):452-6.

44. Alvord LS. Psychological status of patients undergoing electronystagmography. J Am Acad Audiol. 1991;2(4):261-5.

45. Ware JE, Sherbourne CD. The MOS 36-item short-form health survey (SF-36). I. Conceptual framework and item selection. Med Care. 1992;30(6):473-83.

46. Yardley L, Masson E, Verschuur C, Haacke $\mathrm{N}$, Luxon L. Symptoms, anxiety and handicap in dizzy patients: development of the vertigo symptom scale. J Psychosom Res. 1992;36(8):731-41.

47. Kroenke K, Lucas CA, Rosenberg ML, Scherokman BJ. Psychiatric disorders and functional impairment in patients with persistent dizziness. J Gen Intern Med. 1993;8(10):530-5.

48. Sloane PD, Hartman M, Mitchell CM. Psychological factors associated with chronic dizziness in patients aged 60 and older. J Am Geriatr Soc. 1994;42(8):847-52.

49. Mruzek M, Barin K, Nichols DS, Burnett CN, Welling DB. Effects of vestibular rehabilitation and social reinforcement on recovery following ablative vestibular surgery. Laryngoscope. 1995;105(7 Pt 1):686-92.

50. Pardal-Refoyo JL. Investigación de las relaciones y utilidad de la valoración de los factores psicoafectivos en el diagnóstico de procesos supuestamente vestibulares. 1990. Tesis Doctoral. Universidad de Salamanca. 
51. Pardal Refoyo JL, Beltrán Mateos L, Cañizo Alvarez A. Validez de la anamnesis en la evaluación del estado psicoafectivo del enfermo vestibular. An Otorrinolaringol lbero Am. 1994;21(1):85-90.

52. Pardal Refoyo JL, Beltrán Mateos LD. Ansiedad y vértigo. An Otorrinolaringol lbero Am. 1997;24(1):85-95.

53. Pardal-Refoyo JL, Beltrán-Mateos LD. Medición de la intensidad e incapacidad en trastornos del equilibrio mediante escala visual. ORL-DIPS. 1994;20(2):78-81.

54. Monsell EM. New and revised reporting guidelines from the Committee on Hearing and Equilibrium. American Academy of Otolaryngology-Head and Neck Surgery Foundation, Inc. Otolaryngol Head Neck Surg. 1995;113(3):176-8.

55. Cohen HS, Kimball KT, Adams AS. Application of the vestibular disorders activities of daily living scale. Laryngoscope. 2000;110(7):1204-9.

56. Boleas-Aguirre MS, Sánchez-Ferrandiz N, Guillén-Grima $F$, Perez N. Long-term disability of class A patients with Ménière's disease after treatment with intratympanic gentamicin.

Laryngoscope. 2007;117(8):1474-81.
57. Montes-Jovellar L, Guillen-Grima F, PerezFernandez N. Cluster analysis of auditory and vestibular test results in definite Menière's disease. Laryngoscope. 2011 Aug;121(8):1810-7.

58. Alghwiri AA, Whitney SL, Baker CE, Sparto PJ, Marchetti GF, Rogers JC, et al. The Development and Validation of the Vestibular Activities and Participation Measure. Arch Phys Med Rehabil. 2012;93(10):1822-31.

\section{OTROS ENLACES DE INTERÉS}

- BiblioPRO. https://www.bibliopro.org/

- Clasificación Internacional de Deficiencias, Discapacidades y Minusvalías (CIDDM). http://www.un.org/esa/socdev/enable/dpbe19992c.htm

- DeCS. http://decs.bvs.br/cgibin/wxis1660.exe/decsserver/

EMPRO. Evaluating Measures of Patient Reported Outcomes. https://www.bibliopro.org/sobre_empro/index.html

- MeSH. https://www.ncbi.nlm.nih.gov/mesh/

- Vertigo Symptom Scale. https://www.sralab.org/rehabilitationmeasures/vertigo-symptom-scale 


\section{ANEXO 1}

Tabla 3. Términos MeSH y DeCS relativos a vértigo y patología vestibular.

\begin{tabular}{|c|c|c|c|}
\hline $\begin{array}{l}\mathrm{MeSH} \\
\mathrm{DeCS}\end{array}$ & $\begin{array}{l}\text { Término MeSH } \\
\text { https://www.ncbi.nlm.nih.gov/pubmed }\end{array}$ & $\begin{array}{l}\text { Término DeCS } \\
\text { http://decs.bvs.br }\end{array}$ & $\begin{array}{l}\text { Otros térmi- } \\
\text { nos equiva- } \\
\text { lentes }\end{array}$ \\
\hline $\begin{array}{l}\text { Disabled Persons } \\
\text { Personas con } \\
\text { Discapacidad }\end{array}$ & $\begin{array}{l}\text { Persons with physical or mental disa- } \\
\text { bilities that affect or limit their activities } \\
\text { of daily living and that may require } \\
\text { special accommodations. } \\
\text { Year introduced: } 1998(1963)\end{array}$ & $\begin{array}{l}\text { Personas con discapacidades físicas o } \\
\text { mentales que afectan o limitan sus ac- } \\
\text { tividades de la vida diaria y que pueden } \\
\text { requerir adaptaciones especiales }\end{array}$ & Handicapped \\
\hline $\begin{array}{l}\text { Disability Evalua- } \\
\text { tion } \\
\text { Evaluación de la } \\
\text { Discapacidad }\end{array}$ & $\begin{array}{l}\text { Determination of the degree of a } \\
\text { physical, mental, or emotional handi- } \\
\text { cap. The diagnosis is applied to legal } \\
\text { qualification for benefits and income } \\
\text { under disability insurance and to eligi- } \\
\text { bility for Social Security and work- } \\
\text { men's compensation benefits. }\end{array}$ & $\begin{array}{l}\text { Determinación del grado de incapaci- } \\
\text { dad física, mental o emocional. El diag- } \\
\text { nóstico se aplica a la cualificación legal } \\
\text { para la obtención de beneficios e ingre- } \\
\text { sos bajo seguros de incapacidad y la } \\
\text { elegibilidad para el Seguro Social y los } \\
\text { beneficios de compensación de los tra- } \\
\text { bajadores. }\end{array}$ & Handicap \\
\hline $\begin{array}{l}\text { International } \\
\text { Classification of } \\
\text { Functioning, Dis- } \\
\text { ability and Health }\end{array}$ & $\begin{array}{l}\text { The World Health Organization's clas- } \\
\text { sification categories of health and } \\
\text { health-related domains. The Interna- } \\
\text { tional Classification of Functioning, } \\
\text { Disability and Health (ICF) consists of } \\
\text { two lists: a list of body functions and } \\
\text { structure, and a list of domains of ac- } \\
\text { tivity and participation. The ICF also } \\
\text { includes a list of environmental fac- } \\
\text { tors. } \\
\text { Year introduced: } 2014\end{array}$ & $\begin{array}{l}\text { Término no adaptado } \\
\text { Traducción del autor } \\
\text { Clasificación Internacional de Funcio- } \\
\text { namiento, Discapacidad y Salud } \\
\text { Categorías de clasificación de la salud } \\
\text { de la Organización Mundial de la Salud } \\
\text { y dominios relacionados con la salud. } \\
\text { La Clasificación Internacional del Fun- } \\
\text { cionamiento, la Discapacidad y la Sa- } \\
\text { lud (CIF) consta de dos listas: una lista } \\
\text { de funciones y estructura del cuerpo, y } \\
\text { una lista de dominios de actividad y } \\
\text { participación. El ClF también incluye } \\
\text { una lista de factores ambientales. }\end{array}$ & \\
\hline $\begin{array}{l}\text { Vertigo } \\
\text { Vértigo }\end{array}$ & $\begin{array}{l}\text { An illusion of movement, either of the } \\
\text { external world revolving around the } \\
\text { individual or of the individual revolving } \\
\text { in space. Vertigo may be associated } \\
\text { with disorders of the inner ear (EAR, } \\
\text { INNER); VESTIBULAR NERVE; } \\
\text { BRAINSTEM; or CEREBRAL } \\
\text { CORTEX. Lesions in the TEMPORAL } \\
\text { LOBE and PARIETAL LOBE may be } \\
\text { associated with FOCAL SEIZURES } \\
\text { that may feature vertigo as an ictal } \\
\text { manifestation. (From Adams et al., } \\
\text { Principles of Neurology, 6th ed, } \\
\text { pp300-1) }\end{array}$ & $\begin{array}{l}\text { llusión de movimiento, bien del mundo } \\
\text { externo que se mueve alrededor del in- } \\
\text { dividuo o del individuo que se mueve en } \\
\text { el espacio. El vértigo puede estar aso- } \\
\text { ciado con trastornos del OIDO } \\
\text { INTERNO, NERVIO VESTIBULAR, } \\
\text { TRONCO ENCEFÁLICO y CORTEZA } \\
\text { CEREBRAL. Las lesiones en el } \\
\text { LÓBULO TEMPORAL y el LÓBULO } \\
\text { PARIETAL pueden asociarse con } \\
\text { CONVULSIONES focales, que pueden } \\
\text { ocasionar vértigo como manifestación } \\
\text { ictal (Adaptación del original: Adams et } \\
\text { al., Principles of Neurology, 6th ed, } \\
\text { pp300-1). }\end{array}$ & \\
\hline $\begin{array}{l}\text { Dizziness } \\
\text { Mareo }\end{array}$ & $\begin{array}{l}\text { An imprecise term which may refer to } \\
\text { a sense of spatial disorientation, mo- } \\
\text { tion of the environment, or lighthead- } \\
\text { edness. } \\
\text { Year introduced: } 1979\end{array}$ & $\begin{array}{l}\text { Término impreciso que puede referirse } \\
\text { a una sensación de desorientación es- } \\
\text { pacial, movimiento del entorno, o sen- } \\
\text { sación de mareo. }\end{array}$ & \\
\hline $\begin{array}{l}\text { Benign Pa- } \\
\text { roxysmal Positio- } \\
\text { nal Vertigo } \\
\text { Vértigo Posicio- } \\
\text { nal Paroxístico } \\
\text { Benigno }\end{array}$ & $\begin{array}{l}\text { Idiopathic recurrent VERTIGO aSSOCi- } \\
\text { ated wOSITIONAL } \\
\text { NYSTAGMUS. It is associated with a } \\
\text { vestibular loss without other neurolog- } \\
\text { ical or auditory signs. Unlike in } \\
\text { LABYRINTHITIS and VESTIBULAR } \\
\text { NEURONITIS, inflammation in the ear } \\
\text { is not observed. } \\
\text { Year introduced: } 2015\end{array}$ & $\begin{array}{l}\text { VÉRTIGO idiopático recurrente, aso- } \\
\text { ciada a NISTAGMO FISIOLÓGICO. Se } \\
\text { asocia con una pérdida vestibular sin } \\
\text { otros signos neurológicos o auditivos. A } \\
\text { diferencia de LABERINTITIS y } \\
\text { NEURONITIS VESTIBULAR, no se ob- } \\
\text { serva la inflamación en el oído. }\end{array}$ & \\
\hline $\begin{array}{l}\text { Meniere Disease } \\
\text { Enfermedad de } \\
\text { Ménière }\end{array}$ & $\begin{array}{l}\text { A disease of the inner ear } \\
\text { (LABYRINTH) that is characterized by } \\
\text { fluctuating SENSORINEURAL } \\
\text { HEARING LOSS; TINNITUS; epi- } \\
\text { sodic VERTIGO; and aural fullness. It }\end{array}$ & $\begin{array}{lcr}\text { Enfermedad del oído interno } \\
\text { (LABERINTO) } & \text { caracterizada por } \\
\text { HIPOACUSIA NEUROSENSORIAL, } & \text { NEUROSE } \\
\text { ACÚFENOS, VÉRTIGO episódico y }\end{array}$ & \\
\hline
\end{tabular}


INSTRUMENTOS DE EVALUACIÓN DE LA DISCAPACIDAD EN PATOLOGÍA VESTIBULAR PARDAL-REFOYO JL Y BATUECAS-CALETRÍO A

\begin{tabular}{|c|c|c|c|}
\hline $\begin{array}{l}\text { MeSH } \\
\text { DeCS }\end{array}$ & $\begin{array}{l}\text { Término MeSH } \\
\text { https://www.ncbi.nlm.nih.gov/pubmed }\end{array}$ & $\begin{array}{l}\text { Término DeCS } \\
\text { http://decs.bvs.br }\end{array}$ & $\begin{array}{l}\text { Otros térmi- } \\
\text { nos equiva- } \\
\text { lentes }\end{array}$ \\
\hline & $\begin{array}{l}\text { is the most common form of endolym- } \\
\text { phatic hydrops. } \\
\text { Year introduced: } 2008 \text { (1963) }\end{array}$ & $\begin{array}{l}\text { sensación de ocupación del oído afec- } \\
\text { tado. Es la forma más común de hi- } \\
\text { dropesía endolinfática. }\end{array}$ & \\
\hline $\begin{array}{l}\text { Endolymphatic } \\
\text { Hydrops } \\
\text { Hidropesía Endo- } \\
\text { linfática }\end{array}$ & $\begin{array}{l}\text { An accumulation of ENDOLYMPH in } \\
\text { the inner ear (LABYRINTH) leading to } \\
\text { buildup of pressure and distortion of } \\
\text { intralabyrinthine structures, such as } \\
\text { COCHLEA and SEMICIRCULAR } \\
\text { CANALS. It is characterized by } \\
\text { SENSORINEURAL HEARING LOSS; } \\
\text { TINNITUS; and sometimes } \\
\text { VERTIGO. }\end{array}$ & $\begin{array}{l}\text { Acumulación de ENDOLINFA en el } \\
\text { oído interno (LABERINTO) que lleva a } \\
\text { un aumento de presión y distorsión de } \\
\text { las estructuras intralaberínticas, tales } \\
\text { como la CÓCLEA, y los CONDUCTOS } \\
\text { SEMICIRCULARES. Se caracteriza } \\
\text { por HIPOACUSIA } \\
\text { NEUROSENSORIAL, ACÚFENOS y, } \\
\text { en ocasiones,VÉRTIGO. }\end{array}$ & \\
\hline $\begin{array}{l}\text { Vestibular Disea- } \\
\text { ses } \\
\text { Enfermedades } \\
\text { Vestibulares }\end{array}$ & $\begin{array}{l}\text { Pathological processes of the } \\
\text { VESTIBULAR LABYRINTH which } \\
\text { contains part of the balancing appa- } \\
\text { ratus. Patients with vestibular dis- } \\
\text { eases show instability and are at risk } \\
\text { of frequent falls. } \\
\text { Year introduced: } 1990\end{array}$ & $\begin{array}{l}\text { Procesos patológicos del LABERINTO } \\
\text { VESTIBULAR que contiene parte del } \\
\text { aparato del equilibrio. Los pacientes } \\
\text { con enfermedades vestibulares mues- } \\
\text { tran inestabilidad y se hallan en riesgo } \\
\text { de frecuentes caídas. }\end{array}$ & \\
\hline $\begin{array}{l}\text { Vestibulocochlear } \\
\text { Nerve Diseases } \\
\text { Enfermedades } \\
\text { del Nervio Vesti- } \\
\text { bulococlear }\end{array}$ & $\begin{array}{l}\text { Pathological processes of the } \\
\text { VESTIBULOCOCHLEAR NERVE, in- } \\
\text { cluding the branches of COCHLEAR } \\
\text { NERVE and VESTIBULAR NERVE. } \\
\text { Common examples } r \text { are } \\
\text { VESTIBULAR NEURITIS, cochlear } \\
\text { neuritis, and ACOUSTIC NEUROMA. } \\
\text { Clinical signs are varying degree of } \\
\text { HEARING LOSS; VERTIGO; and } \\
\text { TINNITUS. } \\
\text { Year introduced: } 2000(1979)\end{array}$ & $\begin{array}{l}\text { Procesos patológicos del NERVIO } \\
\text { VESTIBULOCOCLEAR, incluidas las } \\
\text { ramas del NERVIO COCLEAR y del } \\
\text { NERVIO VESTIBULAR. Los ejemplos } \\
\text { comunes son NEURONITIS } \\
\text { VESTIBULAR, neuritis coclear y } \\
\text { NEUROMA ACÚSTICO. Los signos clí- } \\
\text { nicos son un grado variable de } \\
\text { PÉRDIDA AUDITIVA, VÉRTIGO y } \\
\text { ACÚFENOS. }\end{array}$ & \\
\hline $\begin{array}{l}\text { Neuroma, Acous- } \\
\text { tic } \\
\text { Neuroma Acús- } \\
\text { tico }\end{array}$ & $\begin{array}{l}\text { A benign SCHWANNOMA of the } \\
\text { eighth cranial nerve } \\
\text { (VESTIBULOCOCHLEAR NERVE), } \\
\text { mostly arising from the vestibular } \\
\text { branch (VESTIBULAR NERVE) dur- } \\
\text { ing the fifth or sixth decade of life. } \\
\text { Clinical manifestations include } \\
\text { HEARING LOSS; HEADACHE; } \\
\text { VERTIGO; TINNITUS; and FACIAL } \\
\text { PAIN. Bilateral acoustic neuromas } \\
\text { are associated } \\
\text { NEUROFIBROMATOSIS 2. (From } \\
\text { Adams et al., Principles of Neurology, } \\
6 \text { 6th ed, p673) }\end{array}$ & $\begin{array}{l}\text { SCHWANNOMA benigno del octavo } \\
\text { par craneal } \\
\text { (NESTIBULOCOCLEAR), la mayoría de } \\
\text { las veces en la rama vestibular } \\
\text { (NERVIO VESTIBULAR), en la quinta o } \\
\text { sexta década de la vida. Las manifes- } \\
\text { taciones clínicas son HIPOACUSIA, } \\
\text { CEFALEA, VÉRTIGO, ACÚFENOS y } \\
\text { DOLOR FACIAL. Los neurinomas } \\
\text { acústicos bilaterales se asocian a } \\
\text { NEUROFIBROMATOSIS TIPO } 2 . \\
\text { (Adams et al., Principles of Neurology, } \\
\text { 6th ed, p673) }\end{array}$ & \\
\hline $\begin{array}{l}\text { Bilateral Ves- } \\
\text { tibulopathy } \\
\text { Vestibulopatía Bi- } \\
\text { lateral }\end{array}$ & $\begin{array}{l}\text { Impairment of the vestibular function } \\
\text { of both inner ears which can cause } \\
\text { difficulties with balance, gait, } \\
\text { VERTIGO, and visual blurring. } \\
\text { Year introduced: } 2017\end{array}$ & $\begin{array}{l}\text { Deterioro de la función vestibular de } \\
\text { ambos oídos internos que pueden cau- } \\
\text { sar dificultades con el equilibrio, } \\
\text { la marcha, VERTIGO y visión borrosa. }\end{array}$ & \\
\hline $\begin{array}{ll}\text { Visual } & \text { Analog } \\
\text { Scale } & \\
\text { Escala } & \text { Visual } \\
\text { Analógica } & \end{array}$ & $\begin{array}{l}\text { A subjective psychometric response } \\
\text { scale used to measure distinct behav- } \\
\text { ioral or physiological phenomena } \\
\text { based on linear numerical gradient or } \\
\text { yes/no alternatives. } \\
\text { Year introduced: } 2014\end{array}$ & $\begin{array}{l}\text { Escala subjetiva de respuestas psico- } \\
\text { métricas que se utiliza para medir fenó- } \\
\text { menos fisiológicos o conductuales, ba- } \\
\text { sada en un gradiente numérico lineal o } \\
\text { alternativas sí/no. }\end{array}$ & \\
\hline $\begin{array}{l}\text { Test Anxiety } \\
\text { Scale } \\
\text { Escala de Ansie- } \\
\text { dad ante Prue- } \\
\text { bas }\end{array}$ & $\begin{array}{l}\text { A self-reporting test consisting of } \\
\text { items concerning fear and worry } \\
\text { about taking tests and physiological } \\
\text { activity, such as heart rate, sweating, } \\
\text { etc., before, during, and after tests. } \\
\text { Year introduced: } 1991(1975\end{array}$ & $\begin{array}{l}\text { Una prueba auto-aplicable compuesta } \\
\text { por elementos sobre miedo y preocu- } \\
\text { pación en relación a situaciones de } \\
\text { prueba y actividades fisiológicas, como } \\
\text { frecuencia cardíaca, sudoración, etc, } \\
\text { antes, durante o después de las prue- } \\
\text { bas. }\end{array}$ & \\
\hline $\begin{array}{l}\text { Brief Psychiatric } \\
\text { Rating Scale }\end{array}$ & $\begin{array}{l}\text { A scale comprising } 18 \text { symptom con- } \\
\text { structs chosen to represent relatively } \\
\text { independent dimensions of manifest }\end{array}$ & $\begin{array}{l}\text { Una escala que consiste en } 18 \text { cons- } \\
\text { tructos de síntomas escogidos para re- } \\
\text { presentar dimensiones relativamente }\end{array}$ & \\
\hline
\end{tabular}

Ediciones Universidad de Salamanca / CC BY-NC-ND [161]

Rev. ORL, 2018, 9, 2, pp. 145-164 
INSTRUMENTOS DE EVALUACIÓN DE LA DISCAPACIDAD EN PATOLOGÍA VESTIBULAR PARDAL-REFOYO JL Y BATUECAS-CALETRÍO A

\begin{tabular}{|c|c|c|c|}
\hline $\begin{array}{l}\text { MeSH } \\
\text { DeCS }\end{array}$ & $\begin{array}{l}\text { Término MeSH } \\
\text { https://www.ncbi.nlm.nih.gov/pubmed }\end{array}$ & $\begin{array}{l}\text { Término DeCS } \\
\text { http://decs.bvs.br }\end{array}$ & $\begin{array}{l}\text { Otros térmi- } \\
\text { nos equiva- } \\
\text { lentes }\end{array}$ \\
\hline $\begin{array}{l}\text { Escalas de Valo- } \\
\text { ración Psiquiá- } \\
\text { trica Breve }\end{array}$ & $\begin{array}{l}\text { psychopathology. The initial intended } \\
\text { use was to provide more efficient as- } \\
\text { sessment of treatment response in } \\
\text { clinical psychopharmacology re- } \\
\text { search; however, the scale was read- } \\
\text { ily adapted to other uses. (From } \\
\text { Hersen, M. and Bellack, A.S., Diction- } \\
\text { ary of Behavioral Assessment Tech- } \\
\text { niques, p. 87) } \\
\text { Year introduced: } 1995\end{array}$ & $\begin{array}{l}\text { independientes de manifiesta. El obje- } \\
\text { tivo inicial era proporcionar una forma } \\
\text { más eficiente de evaluación de la res- } \\
\text { puesta al tratamiento en la investiga- } \\
\text { ción clínica psicofarmacológica. Sin } \\
\text { embargo, la escala fue prontamente } \\
\text { adaptada para otros usos. }\end{array}$ & \\
\hline $\begin{array}{l}\text { Manifest Anxiety } \\
\text { Scale } \\
\text { Escala de Ansie- } \\
\text { dad Manifiesta }\end{array}$ & $\begin{array}{l}\text { True-false questionnaire made up of } \\
\text { items believed to indicate anxiety, in } \\
\text { which the subject answers verbally } \\
\text { the statement that describes him. } \\
\text { Year introduced: } 1991(1975)\end{array}$ & $\begin{array}{l}\text { Cuestionario tipo verdadero-falso com- } \\
\text { puesto de elementos considerados in- } \\
\text { dicativos de ansiedad, en el cual el in- } \\
\text { dividuo indica verbalmente la } \\
\text { afirmación que lo describe. }\end{array}$ & \\
\hline $\begin{array}{l}\text { Surveys and } \\
\text { Questionnaires } \\
\text { Encuestas y } \\
\text { Cuestionarios }\end{array}$ & $\begin{array}{l}\text { Collections of data obtained from vol- } \\
\text { untary subjects. The information usu- } \\
\text { ally takes the form of answers to } \\
\text { questions, or suggestions. } \\
\text { Year introduced: } 2016(1973)\end{array}$ & $\begin{array}{l}\text { Conjunto de datos obtenidos a partir de } \\
\text { individuos voluntarios. La información } \\
\text { usualmente toma la forma de respues- } \\
\text { tas a preguntas, o sugerencias. }\end{array}$ & \\
\hline $\begin{array}{l}\text { Quality of Life } \\
\text { Calidad de Vida }\end{array}$ & $\begin{array}{l}\text { A generic concept reflecting concern } \\
\text { with the modification and enhance- } \\
\text { ment of life attributes, e.g., physical, } \\
\text { political, moral, social environment as } \\
\text { well as health and disease. } \\
\text { Year introduced: } 1977(1975)\end{array}$ & $\begin{array}{l}\text { Concepto genérico que refleja preocu- } \\
\text { pación por la modificación y mejora de } \\
\text { las condiciones de vida, por ejemplo, fí- } \\
\text { sico, político, moral, entorno social, así } \\
\text { como la salud y la enfermedad. }\end{array}$ & \\
\hline
\end{tabular}




\section{ANEXO 2}

Tabla 2. Escalas y cuestionarios utilizados para evaluar la repercusión de la enfermedad vestibular.

\begin{tabular}{|c|c|}
\hline Autor y año & Instrumento \\
\hline Hinchcliffe, 1967 [34] & MMPI (Minnesota Multiphasic Personality Inventory) \\
\hline Brightwell, 1975 [35] & $\begin{array}{l}\text { EPI (Eysenck Personality Inventory) } \\
\text { CMI (Cornell Medical Index) }\end{array}$ \\
\hline Stephens, 1975 [36] & $\begin{array}{l}\text { CCEI (Crown Crisp Experiential Index) } \\
\text { EPI }\end{array}$ \\
\hline Crary, 1977 [37] & $\begin{array}{l}\text { MMPI } \\
\text { TMAS } \\
\text { SSAS } \\
\end{array}$ \\
\hline Rigatelli, 1984 [38] & $\begin{array}{l}\text { ZUNG } \\
\text { SAS } \\
\text { MHQ } \\
\end{array}$ \\
\hline Hallam, 1985 [39] & CCEI \\
\hline Filipo, $1988[40]$ & $\begin{array}{l}\text { SYMPTON CHECK LIST } \\
\text { ADJECTIVE CHECK LIST } \\
\text { STAI (State-Trait Anxiety Inventory) } \\
\text { MMPI }\end{array}$ \\
\hline Coke, r1989 [41] & MMPI \\
\hline Grimm, 1989 [42] & $\begin{array}{l}\text { WAIS } \\
\text { RIQ } \\
\text { WESCHLER MEMORY SCALE } \\
\text { RAY AUDIO VERBAL LEARNING } \\
\text { TRAIL MAKING } \\
\text { SCL-90-R (Symptom Checklist-Revised) } \\
\text { MMPI }\end{array}$ \\
\hline Jacobson, 1990 [13] & DHI (Dizziness Handicap Inventory) \\
\hline McKenna, 1991 [43] & GHQ (General Healt Questionnaire) \\
\hline Alvord, 1991 [44] & STAI \\
\hline Yardley, 1992 [11] & VHQ (Vertigo Handicap Questionnaire) \\
\hline Ware, $1992[45]$ & SF-36 \\
\hline Yardley, $1992[46]$ & $\begin{array}{l}\text { VSS (Vertigo Symptom Scale: two subescales Vertigo scale VSS-VER; Anxiety and Autonomic } \\
\text { symptom VSS-AA) }\end{array}$ \\
\hline Kroenke, 1993 [47] & $\begin{array}{l}\text { Diagnostic Interview Schedule } \\
\text { Sickness Impact Profile }\end{array}$ \\
\hline Sloane, 1994 [48] & SCL-90-R \\
\hline Mruzek1995 [49] & $\mathrm{DHI}$ \\
\hline $\begin{array}{l}\text { Pardal, } 1990[50], \\
1994[51], 1997[52]\end{array}$ & $\begin{array}{l}\text { CET-DE (Cuestionario Estructural Tetradimensional para la Depresión) } \\
\text { STAI } \\
\text { MMPI }\end{array}$ \\
\hline Pardal, 1994 [53] & Escala visual analógica (VAS) \\
\hline $\begin{array}{l}\text { Pardal, } 1998 \text { [32], } \\
1997[33], 2001[5]\end{array}$ & CEMPV (Cuestionario para la Evaluación de la Minusvalía en Patología Vestibular) \\
\hline Honrubia, 1996 [25] & UCLA-DQ (University of California Los Angeles Dizziness Questionnaire) \\
\hline Powell, 1995 [23] & ABC (The Activities-specific Balance Confidence Scale) \\
\hline Monsell, 1995 [54] & AAO-HNS (American Academy of Otolaryngology-Head and Neck Surgery) \\
\hline Jacobson, 1998 [15] & DHI-s (Screening version of the Dizziness Handicap Inventory) \\
\hline Cohen, 2000 [55] [22] & $\begin{array}{l}\text { VADL (Vestibular Disorders Activities of Daily Living Scale) } \\
\text { DHI }\end{array}$ \\
\hline Perez, $2000[21,26]$ & \begin{tabular}{|l|} 
DHI \\
UCLA-DQ \\
Traducción y adaptación transcultural \\
\end{tabular} \\
\hline $\begin{array}{l}\text { Megnigbeto, } 2001 \\
\text { [28] }\end{array}$ & EEV (Evaluation of Vertigo Scale) \\
\hline $\begin{array}{l}\text { Boleas-Aguirre, } 2007 \\
{[56]}\end{array}$ & \begin{tabular}{|l|} 
UCLA-DQ \\
DHI \\
VSS (Vertigo Symptom Scale)
\end{tabular} \\
\hline $\begin{array}{l}\text { Montes-Jovellar Gon- } \\
\text { zalez, } 2011[57]\end{array}$ & $\begin{array}{l}\text { FLS (Functional Level Scale AAO-HNS) } \\
\text { UCLA-DQ } \\
\text { DHI } \\
\text { VSS }\end{array}$ \\
\hline Alghwiri, 2012a [58] & $\begin{array}{l}\text { World Health Organization Disability Assessment Schedule II (WHODAS II) was used to exam- } \\
\text { ine the concurrent validity with the VAP, and the Dizziness Handicap Inventory (DHI) }\end{array}$ \\
\hline
\end{tabular}




\section{ANEXO 3}

Tabla 7. Instrumentos de evaluación de la discapacidad en patología vestibular. Artículos seleccionados.

\begin{tabular}{|c|c|c|}
\hline Autor, año & Método & Principales resultados \\
\hline $\begin{array}{l}\text { Fong, } 2015 \\
{[1]}\end{array}$ & $\begin{array}{l}\text { RS de EC en PubMed, CINAHL y PsycINFO } \\
\text { desde } 1950 \text { hasta } 13 \text { de agosto de 2013. Po- } \\
\text { blación: Ancianos. Idioma: inglés. Objetivo: } \\
\text { identificar las PRO utilizadas en la investiga- } \\
\text { ción vestibular clínica y evaluar sus caracte- } \\
\text { rísticas de prueba y su aplicabilidad para es- } \\
\text { tudiar la pérdida vestibular relacionada con } \\
\text { la edad. Selección: instrumentos con domi- } \\
\text { nios de acuerdo con la CIF. }\end{array}$ & $\begin{array}{l}\text { De } 104 \text { estudios seleccionados se identificaron } 50 \text { PRO } \\
\text { de los cuales los más utilizados fueron 4: } \\
\text { - } \quad \text { Dizziness Handicap Inventory (DHI) } \\
\text { - Activities-specific Balance Confidence (ABC) scale } \\
\text { - Vertigo Symptom Scale (VSS) } \\
\text { - Visual Analogue Scale (VAS). } \\
\text { Ninguno de los instrumentos cumple criterios de evi- } \\
\text { dencia en la aplicación específica en ancianos. }\end{array}$ \\
\hline $\begin{array}{l}\text { Mutlu, } 2013 \\
{[19]}\end{array}$ & $\begin{array}{l}\text { RS en PubMed entre enero de } 1990 \text { y mayo } \\
\text { de } 2012 \text {. } \\
\text { Objetivo: Evaluación de DHI. } \\
\text { Selección de artículos: } 74 \text { artículos seleccio- } \\
\text { nados de } 227 \text {. } \\
\text { Se analizan } 9 \text { puntos. }\end{array}$ & $\begin{array}{l}\text { 1. Confiabilidad, validez y consistencia interna de la } \\
\text { versión original de DHI. } \\
\text { 2. Relación entre pruebas vestibulares y de equilibrio } \\
\text { y DHI. } \\
\text { 3. Asociación entre DHI y las otras escalas relaciona- } \\
\text { das con trastornos de equilibrio. } \\
\text { 4. Análisis factorial exploratorio de DHI. } \\
\text { 5. Versión de screening de DHI (DHI-S). } \\
\text { 6. Traducciones de DHI a diferentes idiomas. } \\
\text { 7. El papel de DHI para evaluar el éxito del trata- } \\
\text { miento de los trastornos del equilibrio. } \\
\text { 8. Los resultados de DHI para diversos trastornos } \\
\text { vestibulares. } \\
\text { 9. Características generales del DHI en pacientes con } \\
\text { deterioro del equilibrio. }\end{array}$ \\
\hline $\begin{array}{l}\text { Alghwiri, } \\
2012[3]\end{array}$ & $\begin{array}{l}\text { Objetivo: Desarrollo y validación del Vestibu- } \\
\text { lar Activities and Participation (VAP) con los } \\
\text { criterios CIF. Método: expertos, método Del- } \\
\text { phi. } \\
\text { Validación: } \\
\text { - Programa de evaluación II de Discapa- } \\
\text { cidad de la Organización Mundial de la } \\
\text { Salud (WHODAS II): } 12 \text { items. } \\
\text { - Dizziness Handicap Inventory DHI: } 25 \\
\text { items: functional, emocional y físico. } \\
\end{array}$ & $\begin{array}{l}\text { VAP Vestibular Activities and Participation } \\
34 \text { items con } 5 \text { niveles (y una respuesta «no contesta»). }\end{array}$ \\
\hline $\begin{array}{l}\text { Duracinsky, } \\
2007[16]\end{array}$ & $\begin{array}{l}\text { RS en Medline y EMBASE. Estudio cualita- } \\
\text { tivo. Artículos publicados en inglés o fran- } \\
\text { cés. Escalas y cuestionarios para evaluar } \\
\text { Quality of life OR handicap. Utiliza una lista } \\
\text { de comprobación para evaluar la calidad de } \\
\text { cada instrumento (ERIQA, European Regu- } \\
\text { latory Issues on Quality of Life Assessment } \\
\text { Working Group). Seleccionan } 29 \text { artículos } \\
\text { en los que se utilizan } 10 \text { cuestionarios. }\end{array}$ & $\begin{array}{l}\text { Handicap or quality of life } \\
\text { - } \quad \text { DHI Dizziness Handicap Inventory } \\
\text { - VADL Vestibular Disorders of Daily Living Scale } \\
\text { - } \quad \text { ABC Activities-specific Balance Confidence } \\
\text { - VHQ Vertigo Handicap Questionnaire } \\
\text { Mixtos } \\
\text { - VDI Vertigo, Dizziness, Imbalance Questionnaire } \\
\text { - UCLA-DQ UCLA Dizziness Questionnaire } \\
\text { - DFI Dizzy Factor Inventory } \\
\text { Evaluación de síntomas } \\
\text { - VSS Vertigo Symptom Scale } \\
\text { - EEV European Evaluation of Vertigo } \\
\text { Específico para la enfermedad de Ménière } \\
\text { - MD-POSI Meniere's Disease Patients-Oriented Se- } \\
\quad \text { verity Index }\end{array}$ \\
\hline $\begin{array}{l}\text { Grill, } 2012 \\
{[20]}\end{array}$ & $\begin{array}{l}\text { RS. Estudio cualitativo } \\
\text { Objetivo: Desarrollo del Brief ICF Core Set } \\
\text { Desarrollar estándares internacionales ICF } \\
\text { para evaluar las funciones que deben me- } \\
\text { dirse en pacientes con vértigo y mareo, en } \\
\text { la investigación y en la práctica clínica para } \\
\text { profesionales de la salud en hospitalizados } \\
\text { o ambulatorios. } \\
\text { Método: } 1 \text {-Estudio transversal, 2-Entrevistas } \\
\text { cualitativas, 3-RS de la literatura, 4-En- } \\
\text { cuesta internacional de expertos }\end{array}$ & $\begin{array}{l}\text { Brief ICF Core Set. } \\
\text { Lista organizada jerárquicamente en } 29 \text { categorías al- } \\
\text { fanuméricas ( } 9 \text { de función corporal, } 4 \text { de estructura cor- } \\
\text { poral, } 10 \text { de actividades y participación y } 6 \text { de factores } \\
\text { ambientales), con niveles crecientes que indican un } \\
\text { mayor grado de detalle. }\end{array}$ \\
\hline
\end{tabular}

PRO: Patient-Reported Outcome (medidas de resultado informadas por el paciente).

ICF: International Clasification Functioning (en español CIF clasificación internacional de la funcionalidad).

RS: revisión sistemática; EC: ensayos clínicos 